Dichlorcarben als Reagens zur Überführung von Carbonsäureamiden in Nitrile

Conversion of Carbonic Acid Amides into Nitriles by Dichlorocarbene

\section{G. HöFLE}

Organisch-Chemisches Institut der Technischen Universität Berlin

(Z. Naturforsch. 28b, 831 [1973]; eingegangen am 8. Juli 1973)

Dehydration, dichlorocarbene, synthesis of nitriles

Eine soeben erschienene Publikation von T. SaRaIE et. al. ${ }^{1}$ über eine neue Nitrilsynthese veranlaßt uns, unsere unabhängigen Ergebnisse auf diesem Gebiet mitzuteilen.

Wie die japanischen Autoren fanden wir, daß Dichlorcarben, nach Makosza ${ }^{2}$ aus Chloroform und Natronlauge erzeugt, primäre Carbonsäureamide in hervorragenden Ausbeuten zu den entsprechenden Nitrilen dehydratisiert.

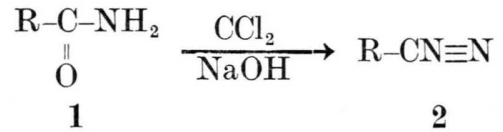

Im Unterschied zu T. SARAIE et al. führen wir die Reaktion bei der Siedetemperatur des Chloroforms mit einem Überschuß an wäßriger Phase durch, wodurch kurze Reaktionszeiten (15-30 Min.) ohne Minderung der Ausbeuten erzielt werden. Bei aromatischen Verbindungen färbt sich das Reaktionsgemisch dabei braun bis schwarz, was aber keinen Einfluß auf die Reinheit der durch Destillation isolierten Nitrile hat.

Die folgende Tabelle gibt einen Überblick über die dargestellten Nitrile und die erhaltenen Ausbeuten.

Während aromatische Carbonsäureamide mit Ausnahme des $p$-Nitrobenzamids durchwegs hohe Ausbeuten ergeben, werden bei aliphatischen Amiden die Ausbeuten durch den Anteil der konkurrierenden Hydrolyse bestimmt. So entsteht Acetonitril nur in Spuren, bei wachsender Kettenlänge bzw. Verzweigung in $a$-Stellung steigen die Ausbeuten jedoch rasch auf über $90 \%$. Aus Bernstein-, Adipinund Phthalsäurediamid konnten keine Dinitrile erhalten werden. Offensichtlich läuft die intramolekular nukleophil katalysierte Hydrolyse ${ }^{3}$ der Wasserabspaltung den Rang ab.

Als Reaktionsmechanismus nehmen wir einen

Sonderdruckanforderungen an Dr. G. HöFle, Organisch-Chemisches Institut der Technischen Universität, D-1000 Berlin 12, Straße des 17. Juni 135.
Tabelle. Nitrile (2) aus Carbonsäureamiden (1)a .

\begin{tabular}{ll}
\hline $1 \mathrm{R}=$ & $\begin{array}{c}2 \text { Ausbeute } \\
{[\%]}\end{array}$ \\
\hline $\mathrm{C}_{6} \mathrm{H}_{5}$ & $85^{\mathrm{b}}$ \\
$4-\mathrm{Cl}_{-} \mathrm{C}_{6} \mathrm{H}_{4}$ & $79^{\mathrm{b}}$ \\
$3-\mathrm{CH}_{3} \mathrm{O}-\mathrm{C}_{6} \mathrm{H}_{5}$ & $72^{\mathrm{b}}$ \\
$4-\mathrm{O}_{2} \mathrm{~N}-\mathrm{C}_{6} \mathrm{H}_{5}$ & $40^{\mathrm{b}}$ \\
$2-\mathrm{Methyl}(3)$-furyl & $80^{\mathrm{b}}$ \\
$\mathrm{C}_{2} \mathrm{H}_{5}$ & $7 \mathrm{c}$ \\
$n-\mathrm{C}_{3} \mathrm{H}_{7}$ & $55^{\mathrm{c}}$ \\
$\left(\mathrm{CH}_{3}\right)_{3} \mathrm{C}$ & $89^{\mathrm{c}}$ \\
$\left(\mathrm{CH}_{3}\right)_{2} \mathrm{C}$ & $94^{\mathrm{c}}$ \\
$\left.\mathrm{CH}_{3}(\mathrm{CH})_{2}\right)_{4}$ & $95^{\mathrm{b}}$ \\
$\mathrm{Cyclohexyl}$ & $95^{\mathrm{b}}$ \\
\hline
\end{tabular}

a Für Reaktionsbedingungen siehe allgemeine Arbeitsvorschrift.

b Isoliert durch Destillation bzw. Sublimation.

c Wird als Gemisch mit Chloroform erhalten, die Ausbeute wurde NMR-spektroskopisch bestimmt, max. Fehler $\pm 5 \%$.

Angriff des Dichlorcarbens auf das Amid-Carbonyl an, worauf nach Übertragung eines Protons Fragmentierung zu Nitril und Formylchlorid eintritt:

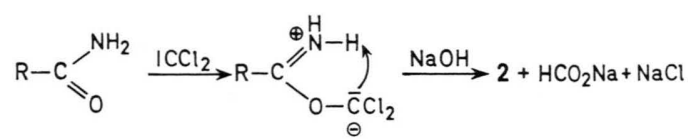

Andere Methoden zur Erzeugung von Dichlorcarben, wie die Thermolyse von Natriumtrichloracetat in Glykoldimethyläther ${ }^{4}$ ergeben unter $\mathrm{Zu}$ satz von Kalium-tert-butoxylat nur 20\% Benzonitril neben Ausgangsmaterial Benzamid.

\section{Allgemeine Arbeitsvorschrift}

Man löst oder suspendiert das Carbonsäureamid $(\mathbf{1} ; 50 \mathrm{mMol})$ in $30 \mathrm{ml}(0,37 \mathrm{Mol})$ Chloroform und $0,5 \mathrm{~g}(2.2 \mathrm{mMol})$ Benzyl-triäthylammonium-chlorid und gibt unter kräftigem Rühren $50 \mathrm{ml}$ warme 40-proz. Natronlauge (0,7 Mol) zu. Nach 15-30 Min. Rückflußerhitzen stoppt man die Reaktion durch Kühlen und Zugabe von mehr Chloroform. Im Falle niedrig siedender Nitrile wird die Chloroformphase zur Entfernung geringer Mengen Triäthylamin, das durch Spaltung des Katalysators entsteht, mit $2 \mathrm{ml}$ 2 N Salzsäure ausgeschüttelt. Anschließend wird mit Magnesiumsulfat getrocknet und das Nitril durch Destillation oder Sublimation isoliert.

1 T. Saraie, T. Ishiguro, K. Kawashima u. K. Morita, Tetrahedron Letters [London] 1973, 2121.

2 M. Makosza u. M. Wawrzyniewicz, Tetrahedron Letters [London] 1969, 4659.

3 T. C. Bruice u. S. J. Benkovic, Bioorganic Mechanisms, Vol. 1, S. 187 und ff., W. A. Benjamin, Inc., New York, Amsterdam 1966.

${ }^{4}$ W. M. Wagner, Proc. Chem. Soc. 1959, 229. 